ARTICLE OPEN

\title{
Early-onset parkinsonism in a pedigree with phosphoglycerate kinase deficiency and a heterozygous carrier: do $P G K-1$ mutations contribute to vulnerability to parkinsonism?
}

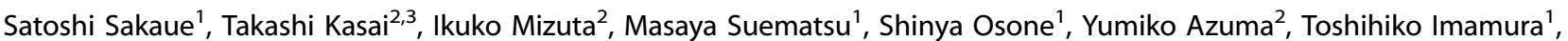
Takahiko Tokuda ${ }^{2,3,4}$, Hitoshi Kanno ${ }^{5}$, Omar M. A. El-Agnaf ${ }^{6}$, Masafumi Morimoto ${ }^{1}$, Masanori Nakagawa ${ }^{2,7}$, Hajime Hosoi ${ }^{1}$ and Toshiki Mizuno ${ }^{2}$

Phosphoglycerate kinase 1 (PGK-1) is a glycolytic enzyme encoded by PGK-1, which maps to the X chromosome. PGK-1 deficiency causes $\mathrm{X}$-linked recessive hereditary chronic hemolytic anemia, myopathy, and neurological disorders due to insufficient ATP regeneration. Early-onset parkinsonism has occasionally been reported as a neurological complication of this condition. However, heterozygous carriers of PGK-1 deficiency were thought to be neurologically asymptomatic. Here, we report a boy with PGK-1 deficiency and his mother, a carrier of a heterozygous mutation in PGK-1, both of whom presented with early-onset parkinsonism. The boy developed parkinsonism at 9 years of age. His parkinsonism partially responded to levodopa treatment. ${ }^{123}$ Imetaiodobenzylguanidine (MIBG) uptake was normal. His mother, who exhibited normal PGK-1 activity in erythrocytes, developed parkinsonism at 36 years of age. Her symptoms were undistinguishable from those of Parkinson's disease (PD), despite her normal uptake of MIBG. Neither a point mutation in nor multiplication of SNCA was found. Additionally, hotspots of $L R R K 2$ and GBA were not mutated. To our knowledge, this report provides the first description of parkinsonism in a carrier of PGK-1 deficiency. Interestingly, PGK-1 is located within the confirmed susceptibility locus for PD known as PARK12. These observations suggest that PGK-1 mutations confer susceptibility to PD.

npj Parkinson's Disease (2017)3:13; doi:10.1038/s41531-017-0014-4

\section{INTRODUCTION}

Phosphoglycerate kinase 1 (PGK-1) is a key enzyme in the glycolytic pathway and is encoded by PGK-1 (Online Mendelian Inheritance in Man (OMIM) \#311800), which maps to the $X$ chromosome and is expressed in all somatic tissues. PGK-1 deficiency (OMIM \#300653) is an uncommon cause of congenital nonspherocytic hemolytic anemia. Clinical features of this deficiency include $\mathrm{X}$-linked recessive chronic hemolytic anemia, myopathy and neurological disorders (e.g., seizures and mental retardation). These phenotypes are diverse among patients due to the differential involvement of three components: erythrocytes; skeletal muscle; and the central nervous system. ${ }^{1}$ Parkinsonism occasionally accompanies PGK-1 deficiency in males. ${ }^{2,3}$ In contrast, heterozygous carriers of this deficiency are generally thought to be asymptomatic. Here, we report a boy with PGK-1 deficiency and his mother, a heterozygous carrier of a PGK-1mutation, both of whom presented with early-onset parkinsonism.

\section{Cases}

The examined family comes from western Japan and includes six living members in three generations, with no history of consanguinity (Fig. 1).
Case 1

(III 1 in Fig. 1). A 16-year-old boy with PGK-1 deficiency developed parkinsonism. We previously reported that this patient was diagnosed with PGK-1 deficiency at 3 years of age based on decreased PGK activity in erythrocytes $(16 \mathrm{IU} / \mathrm{g} \mathrm{Hb}$, normal: $255-325 \mathrm{IU} / \mathrm{g} \mathrm{Hb}$ ) and the novel $P G K-1$ missense mutation c.1060G > C; p.A354P. ${ }^{4}$ (Notably, this mutation was originally described as A353P, which indicates an amino-acid substitution at the 353 rd position from the $\mathrm{NH}_{2}$-terminal serine residue. ${ }^{4}$ ). He had been repeatedly hospitalized for recurrent episodes of myoglobinuria with hemolytic anemia every few months. Moderate intellectual disability had been identified prior to 3 years of age. No epileptic seizures were observed. At 9 years of age, the patient presented with action tremor in his extremities. Rigidity developed at 11 years of age. Therapy with carbidopa/levodopa $25 / 100 \mathrm{mg}$ three times daily produced immediate but partial improvement in the patient's parkinsonism. However, his symptoms gradually progressed. When the patient was 16 years of age, neurological examination revealed severe action tremor in his extremities, especially his left arm, and a mask-like face. Marked rigidity with a dystonic posture was observed in all limbs and the neck. He could no longer walk without assistance. He exhibited

\footnotetext{
${ }^{1}$ Department of Pediatrics, Graduate School of Medical Science, Kyoto Prefectural University of Medicine, Kyoto 602-8566, Japan; ${ }^{2}$ Department of Neurology, Graduate School of Medical Science, Kyoto Prefectural University of Medicine, Kyoto 602-8566, Japan; ${ }^{3}$ AMED-CREST, Japan Agency for Medical Research and Development, Kyoto 602-8566, Japan; ${ }^{4}$ Department of Molecular Pathobiology of the Brain Diseases, Graduate School of Medical Science, Kyoto Prefectural University of Medicine, Kyoto $602-8566$, Japan; ${ }^{5}$ Department of Transfusion Medicine and Cell Processing, Tokyo Women's Medical University, Tokyo 162-8666, Japan; ${ }^{6}$ Neurological Disorders Center, Qatar Biomedical Research Institute

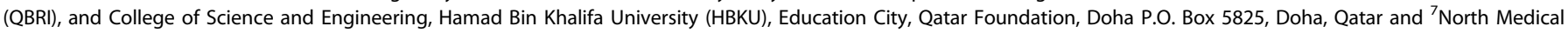
Center, Graduate School of Medical Science, Kyoto Prefectural University of Medicine, Kyoto 602-8566, Japan

Correspondence: Takashi Kasai (kasaita@koto.kpu-m.ac.jp)

Satoshi Sakaue and Takashi Kasai contributed equally to this work.
}

Received: 19 April 2016 Revised: 13 July 2016 Accepted: 14 November 2016

Published online: 31 March 2017 


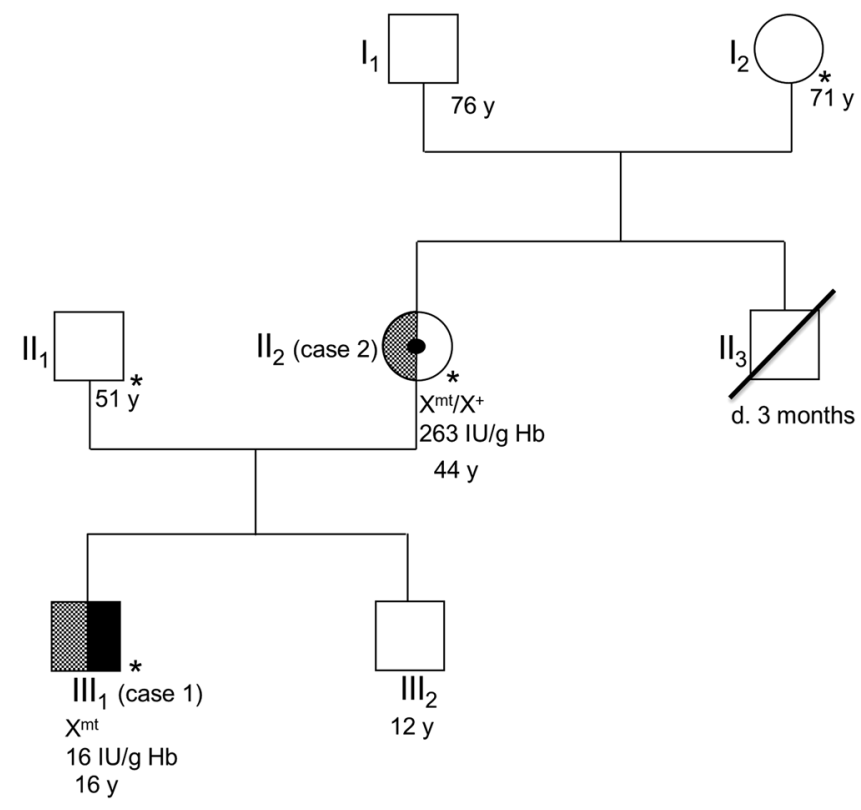

Fig. 1 The patient's pedigree is presented in accordance with standardized human pedigree nomenclature. Solid black indicates the phenotype of classical symptoms of PGK-1 deficiency (i.e., hemolytic anemia and myopathy). The checkerboard pattern indicates the phenotype of parkinsonism. Members with asterisks were neurologically examined by the authors. Cases $1\left(\mathrm{III}_{1}\right)$ and $2\left(\mathrm{II}_{2}\right)$ were also genetically and enzymatically examined. The results of genetic and enzymatic testing for PGK-1 are presented below these cases. $\mathrm{X}^{\mathrm{mt}}$ and $\mathrm{X}^{+}$indicate an allele with the $\mathrm{c} .1060 \mathrm{G}>\mathrm{C}$ mutation and the wild-type allele on the $X$ chromosome, respectively

both urinary and fecal incontinence but no orthostatic hypotension. Brain magnetic resonance imaging revealed mild atrophy of the cerebellum and the basis pontis. Neither the hot cross bun sign nor the putaminal slit sign was identified (Supplementary Fig. 1). A dopamine transporter (DAT) scan using ${ }^{123}$ I-loflupane revealed markedly reduced striatal DAT binding (Fig. 2a). Myocardial imaging with ${ }^{123}$-metaiodobenzylguanidine (MIBG) was normal (heart-to-mediastinum ratio for delayed image: 3.08, institutional normal range: 1.97 to 3.75 ).

\section{Case 2}

$\left(\mathrm{II}_{2}\right.$ in Fig. 1). The mother of case 1 (initially examined for this study at 44 years of age) was a known carrier of the same heterozygous mutation in PGK-1. ${ }^{4}$ At the age of 36 years, she developed resting tremor and a short-stepped gait. Her prior medical history was unremarkable, and she did not have hemolytic anemia or neurologic disorders. PGK-1 activity in her erythrocytes was within the normal range $(263 \mathrm{lU} / \mathrm{g} \mathrm{Hb})$. Neurological examination revealed rigidity in the left upper extremity and both lower extremities, a mask-like face and resting tremor. She had mild fecal incontinence. Neither urinary incontinence nor orthostatic hypotension was observed. Brain computed tomography produced normal findings. Carbidopa/levodopa 25/100 mg three times daily resulted in symptomatic improvement. When the patient was 45 years of age, a DAT scan revealed a right-dominant dopaminergic deficit in the striatum (Fig. 2b). ${ }^{123}$ I-MIBG myocardial imaging was normal (heart-to-mediastinum ratio for delayed image $=2.36$ )

As indicated in Fig. 1, neither a history of hemolytic anemia nor neurological dysfunction, including Parkinson's disease (PD), was identified in the family except for cases 1 and 2 . $\|_{3}$, a brother of $\|_{2}$, died due to cardiac disease at 3 months of age.
To exclude known causes of autosomal dominant parkinsonism, we performed genetic analyses of the coding region and gene dose of SNCA, the hotspot of GBA (exons 5, 6, 8, and 10, including $\mathrm{R} 120 \mathrm{~W}$ and L44P/RecNcil), ${ }^{5}$ and the hotspot of LRRK2 (exons 31, 35 , 41, and 48, including R1441G/H/C, Y1699C, G2019S, I2020T and G2385R). ${ }^{6}$ No mutation was found in either of the aforementioned cases.

\section{DISCUSSION}

Here, we describe a boy with PGK-1 deficiency and his mother, a heterozygous carrier of a PGK-1 mutation, both of whom presented with early-onset parkinsonism. It is unknown whether $\mathrm{II}_{3}$ died from PGK-1 deficiency. Unfortunately, we could not obtain consent for a genetic test from the maternal grandmother of case $1\left(I_{2}\right)$, who appeared to be neurologically normal. Therefore, we have no information regarding whether the mutation in case 2 was inherited from the patient's mother or was a de novo mutation. Neurological symptoms of the boy in case 1 included parkinsonism and were accompanied by cerebellar atrophy, autonomic dysfunction, and normal MIBG uptake. These symptoms may be similar to those of multiple system atrophyparkinsonism rather than PD. Symptoms exhibited by this boy's mother included levodopa-responsive parkinsonism with resting tremor and were consistent with clinical diagnostic criteria for PD, despite her normal uptake of MIBG. To our knowledge, this report provides the first evidence that parkinsonism can develop not only in a patient with PGK-1 deficiency but also in a heterozygous carrier of a PGK-1 mutation without an enzymatic deficiency. We did not comprehensively exclude all known genetic abnormalities related to susceptibility to parkinsonian syndromes, including PD. However, our cases appear unlikely to be caused by known parkinsonism-related mutations for the following reasons. First, parkinsonism that develops in patients under the age of 10 years is quite unusual for known dominantly inherited forms of PD. Second, in our cases, parkinsonism was more severe and exhibited an earlier age of onset in the child compared with the mother. The appearance of more severe symptoms at earlier ages in successive generations has not been reported in known hereditary parkinsonian syndromes, whereas this phenomenon is easy to understand when we consider differences in gene dosages of $P G K-1$ between males and females.

According to a current database (OMIM \#311800), PGK-1 lies on the $X$ chromosome at $78,065,188-78,129,296$, a region within Xq21.1, although $P G K-1$ was originally reported to map to chromosome Xq13. ${ }^{7}$ Interestingly, the region between Xq21 and q25 is known as a susceptibility locus for classical PD associated with PARK12 (OMIM \#300557), and the causative gene for this disease has not been identified. ${ }^{8,9}$ We therefore speculate that mutations in PGK-1 may contribute to the pathogenesis of PARK12-associated PD.

Parkinsonism in patients with PGK-1 deficiency has been postulated to occur due to insufficient ATP regeneration in the substantia nigra as a result of low levels of PGK activity. ${ }^{2}$ In heterozygous carriers, the mutant allele of PGK-1 on the $\mathrm{X}$ chromosome is randomly inactivated during early embryonic stages due to lyonization, resulting in a "mosaic" or "patchy" pattern of enzymatic activity. Therefore, selective enzymatic deficiency in the substantia nigra is possible in heterozygous carriers even when erythrocytes exhibit normal enzymatic activity, as observed in the present case. Based on this hypothesis, the penetrance of parkinsonism in PGK-1 deficiency is expected to be incomplete since variability in lyonization would lead to considerable variability in the severity of parkinsonism in female carriers.

Several studies have established an association between heterozygous mutations in GBA, which is responsible for Gaucher disease, and PD. These findings have induced a paradigm shift in the PD field from the "common disease: common variant" 


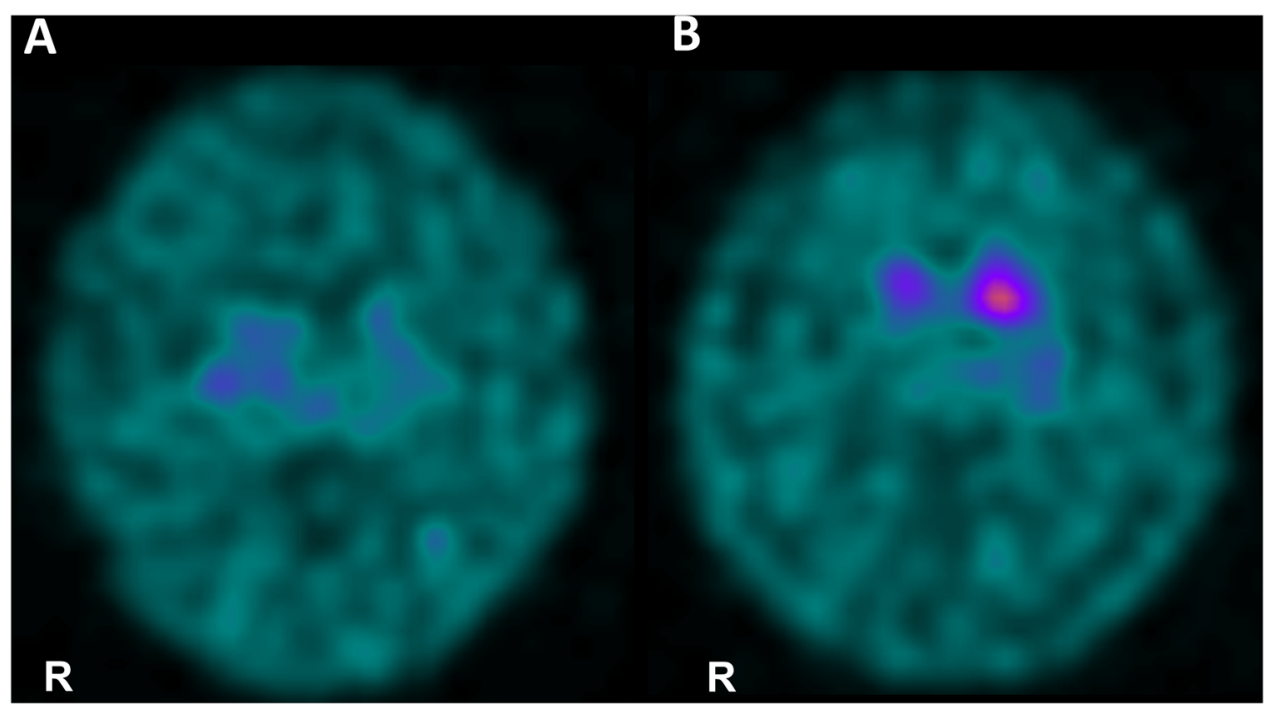

Fig. 2 DAT images for case 1 (a) and case 2 (b) are presented. Both images reveal decreased DAT uptake in the striatum. Specific binding ratios were semiquantitatively calculated using DaT View software (Nihon Medi-Physics, Tokyo, Japan) based on Bolt's method. ${ }^{10}$ The right and left specific binding ratios were 1.87 and 1.71 , respectively, in (a) and 2.50 and 4.19 , respectively, in (b). The reported cut-off value was 4.5 . $^{10}$

hypothesis to the "common disease: multiple rare variant" hypothesis. ${ }^{5}$ We may have encountered a similar situation in this family. Neurologists and pediatricians should therefore look carefully for parkinsonism in not only PGK-1 deficiency patients but also carriers of this deficiency. Further studies are needed to clarify whether multiple rare variants of $P G K-1$ confer susceptibility to PD or other parkinsonian syndromes.

\section{ACKNOWLEDGEMENTS}

This work was supported by AMED, AMED-CREST (to T.T.), and a Grant-in-Aid (No. 15K09319) from the Ministry of Education, Culture, Sports, Science and Technology of Japan (to T.K.).

\section{AUTHOR CONTRIBUTIONS}

S.S. and T.K.: study concept and design; S.S., T.K., M.S., S.O., T.I. and Y.A.: collection of clinical information; I.M., T.I., and M.M.: genetic testing; H.K.: Enzymatic testing; T.T., O.E. and M.N.: critical revision of the manuscript for important intellectual content; $H$. H. and T.M.: study supervision.

\section{COMPETING INTERESTS}

T.K. has received research support from a Grant-in-Aid (No. 15K09319) from the Ministry of Education, Culture, Sports, Science and Technology of Japan and from AMED and AMED-CREST. T.T. has received research support from AMED and AMEDCREST. O.M.A.E-A. has received research support from The Michael J. Fox Foundation for Parkinson's Research (NY). The remaining authors declare no competing interests.

\section{REFERENCES}

1. Beutler, E. PGK deficiency. Br. J. Haematol. 136, 3-11 (2007).

2. Sotiriou, E., Greene, P., Krishna, S., Hirano, M. \& DiMauro, S. Myopathy and parkinsonism in phosphoglycerate kinase deficiency. Muscle Nerve 41, 707-710 (2010).
3. Konrad, P. N., McCarthy, D. J., Mauer, A. M., Valentine, W. N. \& Paglia, D. E. Erythrocyte and leukocyte phosphoglycerate kinase deficiency with neurologic disease. J. Pediatr. 82, 456-460 (1973).

4. Morimoto, A. et al. A novel missense mutation (1060G -->C) in the phosphoglycerate kinase gene in a Japanese boy with chronic haemolytic anaemia, developmental delay and rhabdomyolysis. Br. J. Haematol. 122, 1009-1013 (2003).

5. Mitsui, J. et al. Mutations for Gaucher disease confer high susceptibility to Parkinson disease. Arch. Neurol. 66, 571-576 (2009).

6. Rudenko, I. N. \& Cookson, M. R. Heterogeneity of leucine-rich repeat kinase 2 mutations: genetics, mechanisms and therapeutic implications. Neurotherapeutics 11, 738-750 (2014).

7. Willard, H. F., Goss, S. J., Holmes, M. T. \& Munroe, D. L. Regional localization of the phosphoglycerate kinase gene and pseudogene on the human $\mathrm{X}$ chromosome and assignment of a related DNA sequence to chromosome 19. Hum. Genet. 71, 138-143 (1985).

8. Pankratz, N. et al. Genome-wide linkage analysis and evidence of gene-by-gene interactions in a sample of 362 multiplex Parkinson disease families. Hum. Mol. Genet. 12, 2599-2608 (2003).

9. Hardy, J., Cai, H., Cookson, M. R., Gwinn-Hardy, K. \& Singleton, A. Genetics of Parkinson's disease and parkinsonism. Ann. Neurol. 60, 389-398 (2006).

10. Tossici-Bolt, L., Hoffmann, S. M., Kemp, P. M., Mehta, R. L. \& Fleming, J. S. Quantification of [123I]FP-CIT SPECT brain images: an accurate technique for measurement of the specific binding ratio. Eur. J. Nucl. Med. Mol. Imaging 33, 1491-1499 (2006).

(i) This work is licensed under a Creative Commons Attribution 4.0 International License. The images or other third party material in this article are included in the article's Creative Commons license, unless indicated otherwise in the credit line; if the material is not included under the Creative Commons license, users will need to obtain permission from the license holder to reproduce the material. To view a copy of this license, visit http://creativecommons.org/licenses/by/ $4.0 /$

(c) The Author(s) 2017

Supplementary Information accompanies the paper on the npj Parkinson's Disease website (doi:10.1038/s41531-017-0014-4). 